\title{
Risk of SARS-CoV-2 Transmission from Humans to Pets and Vice Versa
}

\author{
Zameer Shervani, Intazam Khan, Noha Yamin Siddiqui, Tooba Khan, and Umair Yaqub Qazi
}

\section{ABSTRACT}

Numerous studies have described human-to-human airborne and contactbased transmissions of SARS-CoV-2, the epidemiological agent of coronavirus disease 2019 (COVID-19). In this review article, we assess the COVID-19 reverse-zoonotic transmission from humans-to-household pets and zoonotic transmission from pets-to-humans, and animal-to-animal transmission between cohoused pets including cats, dogs, and ferrets. COVID-19 in mink, ducks, pigs, chickens, and ectoparasite of domestic pets such as cat flea are also discussed. Cats and ferrets were found highly susceptible to SARS-COV-2; dogs susceptible to a lower degree; and chickens, pigs, and ducks not susceptible. Reverse zoonotic SARS-COV-2 transmission in dogs and cats was found as confirmed by genetic analysis of virus strains isolated from pets and pet owners. Most studies show absence of clinical symptoms in infected dogs and cats. There is no evidence of zoonotic transmission of SARS-COV-2 from pets, although the possibility could not be ruled out. COVID-19 in mink farms showed both human-tomink and subsequent mink-to-human (zoonotic) transmission raising concerns about mink becoming possible unexpected intermediate hosts of SARS-COV-2. Preventative and control measures limiting zoonotic and reverse zoonotic COVID-19 transmissions should be developed and enforced publicly to minimize virus spread. Our article is important for animal and pet management in COVID-19 pandemic. As there is no evidence of SARS-CoV-2 transmission from household pets to humans, the pets must not be abandoned. To avoid virus transmission from infected households and pet-to-pet, the pets must be quarantined and isolated similar to humans. Leaving pets roaming in the community will increase the chances of virus spread. The original source of the virus is still unknown therefore, pets and animal management is necessary to stop the current and future pandemic.

Keywords: SARS-CoV-2, COVID-19, Zoonotic COVID-19 Transmission, COVID-19 and Pets, SARS-CoV-2 in Mink.
Published Online: January 30, 2021

ISSN: 2593-8339

DOI: $10.24018 /$ ejmed.2021.3.1.684

\section{Zameer Shervani*}

Food \& Energy Security Research \& Product Centre, Sendai, Japan

(e-mail: shervani.nanotek@gmail.com) Intazam Khan

Department of Neurology, North Shore

University Hospital, New York, U.S.A.

Noha Yamin Siddiqui

Food \& Energy Security Research \& Product Centre, Sendai, Japan

Tooba Khan

Food \& Energy Security Research \& Product Centre, Sendai, Japan

Umair Yaqub Qazi

Chemistry Department, College of Science, University of Hafr Al Batin, Hafr Al Batin, K.S.A.

*Corresponding Author

\section{INTRODUCTION}

In the previous articles [1]-[3], the origin of COVID-19 pandemic, advances in testing and treatment, and vaccine development have been described. The COVID-19 pandemic remains a global concern as the daily death toll continues to rise. The number of cases in the most populated country India has declined in the past few days, although the current caseload is worrisome. The COVID-19 caseload in India surpassed 7.5 million on October 18, 2020. India recorded a spike of $>90,000$ cases per day during September 5-19 and reached 97, 859 on September 16. On September 17, cases dropped to 96, 793 and a continuous decline has occurred since then. For example, on October 18 , there were 55,511 cases. Cases in India are receding in the first wave of infection. The 4-to-5 million, 5-to-6 million, and 6-to-7 million jumps occurred in 11, 12, and 13 days, respectively. After the US, India is the only country to reach 7.5 million COVID-19 cases [4]-[9]. The global COVID-19 cases reached above 40 million on October 19, 2020 [10]. In October, during the second wave of infection, a record number of daily infections was recorded in Germany, France, Italy, and other parts of Europe and Russia. There is also a resurgence of infection in the US. The countries can be arranged according to the number of cases: US (8.1 million) > India (7.5 million) > Brazil (5.2 million) > Russia (1.4 million). The pandemic has caused $>1.1$ million global deaths already. It took 166 days to reach the figure of 10 million infections since the occurrence of the first case. The first 10 million cases were recorded in January-June. The number of days the pandemic took to reach subsequent 10 million cases are: 44, 38, 31 days for 20, 30, and 40 million cases, respectively. Currently, the global viral caseload is 40 million with $>1.1$ million deaths. Moreover, the second wave of infection has hit Europe and 
the USA. In this grim situation, it is of utmost necessary to study the nature of the COVID-19 virus and take all the precautionary measures to stop the spread of the pandemic.

In this review article, we assess the possibility of pets as the intermediate host for virus transmission from humans to pets and back from pets to humans. Based on the number of observations that accidentally occurred and experiments conducted on pets, we reached the conclusion that there is no evidence that if pets are the source of infection. But the possibility of pets can infect humans and cohoused pets have also not been ruled out. The management of infected and healthy pets have also been suggested.

\section{RESULTS AND DISCUSSION}

\section{A. COVID-19 Virus in Domestic Pets}

As the airborne nature of coronavirus has been established [11], it is necessary to investigate the possibility of the virus transmission in animals when they come in contact with humans or fellow animals. The study is useful for animal management during the pandemic and also beneficial in controlling the disease. As the intermediate source of SARS-CoV-2 virus is unknown, the susceptibility of animals (dogs, pigs, cats, chickens, ferrets, and ducks) which are in close contact with humans has been studied for coronavirus transmission and multiplication. The study [12] showed that among cats, dogs, pigs, chickens, ducks, and ferrets, only cats and ferrets were found highly susceptible to airborne SARS-CoV-2 infection. The coronavirus replicates poorly in dogs and does not replicate in pigs, chickens, and ducks. But ferrets and cats are susceptible to the virus. The RNA multiplication and titers were measured in above listed animals after inoculation of the virus. The distribution of multiplied RNA was investigated in different organs of ferrets and cats. Large titers $\left(10^{10}\right.$ viral RNA (copies/g)) were found in the nasal turbinate of inoculated ferrets. The soft palate and tonsils (upper respiratory system) also had a significant amount of virus. The RNA was detected on day $2,4,6$, and 8 of post inoculation in ferrets. Antibodies against SARS-CoV-2 were detected in all ferrets. The virus multiplication though did not cause severe disease or death in ferrets. Cats were inoculated with the SARS-CoV-2 virus and RNA replication was studied. Viral RNA was detected in the nasal turbinate, soft palates, tonsils, tracheas, lungs, and small intestine of inoculated cats. The detected viral titers was as high as $10^{10}$ RNA (copies/g) in nasal turbinate. In conclusion, ferrets and cats are highly susceptible, while dogs have low susceptibility and chickens, ducks, and pigs are not susceptible to the virus.

In New York (US), two pet cats were tested [13] positive for COVID-19. This was the first case of household animals affected by the pandemic. The cats acquired the infection from infected members in the households or neighbourhoods. The cats were symptomatic for mild respiratory sickness. Tigers and lions (Felidae or big cat family) in Bronx Zoo (New York) were also tested positive for the virus [13]. The animals in a zoo are in frequent contact with the humans that has infected them. Thus, virus transmission from humans to animals was established. There is no evidence if the pets can transfer the virus to humans although the possibility has not been ruled out. Therefore, it is recommended [1] to keep pets away from interacting with other pets and humans. Investigation [14] confirmed the spread of airborne SARS-CoV-2 virus among cats. For this reason, limiting the contact of pet cats from humans and other cats has been recommended. In a mink farm located in Spain, $87 \%$ of the mink and 7 farm employees were found infected [15] with the COVID-19 virus. One hundred thousand mink were culled in Spain and similar culling of one million mink was done on a Dutch mink farm. The spread of the SARS-CoV-2 virus, on mink farms in Europe, affecting the mink and farm workers caused panic in farming industries. The officials have suspected the virus spread in mink through an infected farm worker via reverse zoonosis (humans-to-animals transmission). The possibility of zoonotic (animals-to-humans) transmission of the virus from mink to human should be investigated.

Cats living with the owner's family of several members affected [16] by the COVID-19 virus were investigated for infection and virus transmission. One of the two cats' clinical condition deteriorated but it was not confirmed if the SARS-CoV-2 virus caused the symptoms as the cat had preexisting medical distress. Histopathological lesions compatible with a viral infection were not detected in the post mortem analysis, although the SARS-CoV-2 RNA was detected in organs of the cat. Reverse zoonosis (human-tocat transmission) was established in this case as the cat was seen to develop infection post contact with COVID-19 infected owners. The viral genome isolated from the cat was very identical to the viral genetic sequence isolated from the owner. The second cat living with the infected cat had no clinical symptoms. Both the cats had SARS-CoV-2 neutralizing antibodies indicated the presence of productive viral infection which came from infected owners. Experiments were conducted [17] to verify the transmission of virus from SARS-CoV-2 inoculated cat to cohoused healthy cats. Three cats were inoculated with the SARSCoV-2 virus, then each inoculated cat was cohoused with one healthy cat to ascertain the virus transmission between the cats. On day 1 , the virus was found in the nasal swab of two inoculated cats. The third incubated cat was found carrying the virus on day 3 . The virus was detected in cats till day 5 and 6 . On day one, the healthy cats were cohoused with infected cats. After 2 to 5 days, the virus was spread in all the cohoused cats. The virus titers in the cohoused cats reached the maximum of $4.5 \log _{10}$ PFU (plaque-forming units) per millilitres. The virus shedding lasted for 4-5 days. All the inoculated and cohoused cats remained asymptomatic for body temperature, weight loss, and conjunctivitis. In all the cats, IgG antibody titers was in the range 1:5120-1:20480; detected on day 24 after inoculation. There is a potential risk of human-cat-human transmission similar to the human-human transmission in closed spaces. Here the cats are acting as silent spreader hosts. The virus was found transmitted from humans to these animals. Also, the virus can spread from one infected animal to other healthy animals. Infected pet must be isolated from other healthy pets. The virus in these animals though has not caused any symptoms. The finding [18] showed that the cats were susceptible to SARS-CoV-2 infection though the cats 
did not show clinical symptoms and the viral shedding lasted for 5-6 days. The immune responses developed in the cats prevented the reinfection. The observations of viral shedding and immunity development are helpful in vaccine preparation for both humans and animals.

Setting free the domestic cats in the community was investigated [19] using computer simulation from the viewpoint of COVID-19 transmission. Simulations were performed when a number of infected or healthy cats were set free in a small community and the number of infected persons was recorded. More people were infected when more cats were set free in the community suggested that cats must not be abandoned for the fear of spreading the virus in the household. If cats are set free, it will cause more virus spread. Therefore, it is advised to quarantine or isolate the cats at home from an infected household member or community rather than abandoning them.

The presence of SARS-CoV (coronavirus in general) virus derived and cell receptor angiotensin-converting enzyme (ACE) RNA and proteins were detected in domestic cat flea (Ctenocephalides felis, C.felis) [20]. The domestic cat flea $(C . f e l i s)$ are abundant ectoparasite infesting humans, companion animals, and wildlife. Similar to SARS-CoV presence, the cat flea may be acting as the reservoir of SARS-CoV-2 that should be studied further. The cat flea may act as SARS-CoV-2 domestic hosts to spread the COVID-19 disease by acting as biological or mechanical vectors. In the study, though SARS-CoV-2 was not identified because of experimental limitations but possibility cannot be ruled out. Moreover, the identification of coronavirus-derived RNA in the midgut of an unfed cat flea suggested the possibility of virus infection and replication in fleas. Although current evidence suggests that pets are probably dead-end-hosts with the small risk of transmission to humans. Though, it is suggested that cat flea may act as biological or mechanical vector of SARS-CoV-2. The ACE2 (angiotensin-converting enzyme 2) protein sequence of macaque (Macaca mulatta) and chimpanzee (Pan troglodytes) are the closest to human ACE2 with 94.9 and 99\% sequence identity [21], respectively. The domestic cat (Felis catus) is the next most closely $(85.2 \%$ ) related ACE2 sequence to the human. Because of much ACE2 sequence resemblance in domestic cats with humans compared to other pets, cats are more susceptible to the SARS-CoV-2 virus among all the mammalian pet species. The SARSCoV-2 spike receptor binds to ACE2 receptor causes the disease thus cats are affected by the pandemic most than other pets.

Two pet dogs became infected [22] with coronavirus from their coronavirus positive owners in Japan. Dogs remained asymptomatic and later one of the dogs shed the virus and became negative. Whether the virus belonged to the owners' body or produced in dogs was not verified. To prove if the virus can multiply in the dog's body more tests are needed. The persons or animals in contact with the dogs did not show any symptoms. The pet dogs and cats can be the source of human infection has been denied but precaution is necessary as suggested above. In a COVID-19 affected family, 2 out of 15 dogs were found infected [23] with SARS-CoV-2 virus. The SARS-CoV-2 was detected for 13 days from one of the dogs as determined by RT-PCR.
Antibody responses were confirmed in both the dogs using plaque-reduction-neutralizing assays. The viral genetic sequences in both the dogs were identical to the virus of the host family, confirming human to dog transmission. Both the dogs remained asymptomatic. A low viral load in the dog was noticed in the range $7.5 \times 10^{2}-2.6 \times 10^{4}$ RNA copies per $\mathrm{ml}$ of specimen. Due to the low viral load, virus cultivation was not possible. For COVID-19 virus cultivation success in human, the viral load must be more than $10^{6}$ per $\mathrm{ml}$. The human receptor ACE2 and canine ACE2 behave similarly, the binding of ACE2 of both human and canine with SARS-CoV-2 spike proteins occurs. The virus has not caused illness in dogs though they shed the virus. If the dog can transmit the virus to other dogs or animals and back to the human is not known yet. The second dog in the household did not get the virus therefore, the virus was not transmitted between the dogs sharing the household. The virus is not circulating in animals: dogs, cats, and horses. Cat-to-cat transmission has been established though.

\section{B. COVID-19 Virus in Mink}

In a recent report [24], it was first confirmed that the SARS-CoV-2 virus transmitted from COVID-19 affected mink farm workers to mink. After the virus was introduced to mink by humans, it was subsequently spilled to a large mink population. The workers and mink when inhaling contaminated air and dust became infected with the virus. This was the first zoonosis event noticed. Farm workers had the symptoms of COVID-19 before the signs of the disease appeared in mink. The observation suggested the need for the prevention of virus-spread in other farms and human surroundings. Genome sequencing has been used [25] to explain the SARS-CoV-2 outbreaks on 16 mink farms between humans and mink and back to humans. Mink were infected by humans living and working on these farms in Netherlands. The virus evolved after initially introduced from humans. It was widely spread and circulated for several weeks among mink on these 16 farms. On detection and analysis, $68 \%$ of farm residents, employees, and contacts were found infected with the virus. The type of virus strain detected in humans had a genomic sequence signature similar to the animal providing evidence of reverse animal to human transmission had occurred in mink farm. The observation suggested that animals were the source of human infection. Mink and animal species similar to mink are the reservoir of SARS-CoV-2. Animal management is needed to stop the SARS-CoV-2 outbreak when mink farms are the big source of the virus and strong viral mutation. The viral mutation occurring in mink is a more challenging task for treatment and vaccine development. Strict biosecurity measures are needed to prevent virus transmission and spread from humans to mink and back to humans. This will enable to stop spread and virus mutation.

\section{CONCLUSION}

Cats and ferrets were found highly susceptible to SARSCoV-2; dogs susceptible to a lower degree; and chickens, pigs, and ducks not susceptible to infection. SARS-CoV-2 replicates exclusively in the upper respiratory tract of ferrets 
where an abundance of ACE2 is found. Thus, ferrets may be a suitable animal model for evaluating antiviral treatments against COVID-19 given that SARS-CoV-2 uses ACE2 as the cell-entry receptor during human infection. Numerous studies have provided evidence for reverse zoonotic SARSCoV-2 transmission in cats and dogs as confirmed via the high genetic similarity of virus strains found in pets and infected pet owners. Airborne transmission can occur between cats attributed to the efficient replication of SARSCoV-2 in cats. Studies attributed death of cats to preexisting illnesses rather than COVID-19. Cats produce SARS-CoV-2 neutralizing antibodies and remain seropositive during infection indicating the presence of productive viral infection. Thus, cats may be used as a potential animal model for COVID-19 studies assessing transmission routes, virus replication, and the efficacy of new treatments. Dogs display significantly small RNA titers and dog-dog transmission has not been observed. There is no evidence that SARS-CoV-2 causes illness in dogs and cats as clinical symptoms remain absent. The asymptomatic nature of viral infection in pets may enhance the spread of COVID-19 disease "silently" or unpredictably within the community. There is no evidence of zoonotic transmission of SARS-CoV-2 from household pets, although the possibility should not be ruled out. Mink-to-humans zoonotic transmission of COVID-19 has been observed in mink farms raising concerns about mink farms becoming possible unexpected intermediate hosts of SARS-CoV-2. Further studies should assess the potential risk of developing new SARS-CoV-2 variants as the virus mutates in the intermediate host mink populations. The study showed that C. felis or Cat Flea (ectoparasites of domestic cats) can be the reservoir for SARS-CoV-2 and the biological vector for the virus. Cat fleas can also spread the virus mechanically. It is necessary to observe the pets of infected owners for SARS-CoV-2 infection. Preventative measures limiting human-to-pet and vice versa COVID-19 transmissions should be developed and enforced publicly to limit virus spread. Managing the pets is not abandoning them, as virusspread will be promoted if pets are set free roaming in a neighbourhood. The advisable strategy is observation, quarantine, isolation, and testing the pets for the virus.

\section{ACKNOWLEDGMENT}

Zameer Shervani (corresponding author) dedicated this COVID-19 research to his grandfather Inayat Ahmad Khan of Kanobi (Aligarh, India).

\section{STATEMENT OF HUMAN AND ANIMAL RIGHTS}

No animal and human rights issues exist in this research. No animal or human experiments were conducted in this research.

\section{AUTHORS' DECLARATION}

The data and results given in this article on the COVID19 pandemic and virus are very reproducible. The authors take responsibility for the authenticity of the data. Authors
Noha Yamin Siddiqui and Tooba Khan contributed remotely.

\section{REFERENCES}

[1] Zameer Shervani, Intazam Khan, Tasrina Khan, Umair Yaqub Qazi, COVID-19 Vaccine, Advances in Infectious Diseases, 2020, 10, 195 210 .

[2] Zameer Shervani, Intazam Khan, Umair Yaqub Qazi, SARS-Cov-2 Delayed Tokyo 2020 Olympics: Very Recent Advances in COVID-19 Detection, Treatment, and Vaccine Development Useful Conducting the Games in 2021, Advances in Infectious Diseases, 2020, 10, 56-66.

[3] Zameer Shervani, Intazam Khan, Tasrina Khan, Umair Yaqub Qazi, World's Fastest Supercomputer Picks COVID-19 Drug, Advances in Infectious Diseases, 2020, 10, 211-225.

[4] Covid cases top 75 lakh as slowdown continues, The Times of India October 19, 2020

[5] India's active caseload of Covid-19 decreases but fear of resurgence remains, Hindustan Times, October 11, 2020.

[6] TOI Coronavirus Live Tracker: How India is fighting coronavirus. Updates, myth-busters, tips and more. (n.d.). Retrieved from https://timesofindia.indiatimes.com/coronavirus

[7] India drops to No.2 in daily Covid cases as US surges again, The Times of India, October 18, 2020.

[8] https://www.worldometers.info/coronavirus/country/india/

[9] India's Covid cases now at 6 million, last $1 \mathrm{~m}$ rise takes day more than previous, The Times of India, September 28, 2020.

[10] Global coronavirus cases top 40 mil., NHK WORLD-JAPAN, October 20, 2020.

[11] Zameer Shervani, Intazam Khan, Noha Yamin Siddiqui, Tooba Khan, Umair Yaqub Qazi, Viability of SARS-CoV-2 and Sanitization Methods, European Journal of Medical and Health Sciences, 3(2021)22-27.

[12] Shi, J.Z., Wen, Z.Y., Zhong, G.X., Yang, H.L., Wang, C., Huang, B.Y., Liu, R.Q., He, X.J., Shuai, L., Sun, Z.R., Zhao, Y.B., Liu, P.P., Liang, L.B., Cui, P.F., Wang, J.L., Zhang, X.F., Guan, Y.T., Tan, W.J., Wu, G.Z., Chen, H.L. and Bu, Z.G. (2020) Susceptibility of Ferrets, Cats, Dogs, and Other Domesticated Animals to SARS-Coro$\begin{array}{llll}\text { navirus } & 2 . & \text { Science, } & 368,\end{array}$ https://doi.org/10.1126/science.abb7015.

[13] 2 cats in NY become first US pets to test positive for virus, The Mainichi, April 23, 2020.

[14] Abe, A., Study Shows COVID-19 Can Spread Easily between Cats. The Asahi Shimbun, June 7, 2020.

[15] A Million Mink Culled in Netherlands and Spain amid COVID-19 Fur Farming Havoc. The Guardian, 17 July 2020.

[16] Joaquim Segalés, Mariona Puig, Jordi Rodon, Carlos Avila-Nieto, Jorge Carrillo, Guillermo Cantero, Maria Teresa Terrón, Sílvia Cruz, Mariona Parera, Marc Noguera-Julián, Nuria Izquierdo-Useros, Víctor Guallar, Enric Vidal, Alfonso Valencia, Ignacio Blanco, Julià Blanco, Bonaventura Clotet, and Júlia Vergara-Alert, Detection of SARS-CoV-2 in a cat owned by a COVID-19-affected patient in Spain, Science, 117, 2020, 24790-24793.

[17] P. J. Halfmann et al., Transmission of SARS-CoV-2 in domestic cats. N. Engl. J. Med. 383, 592-594 (2020).

[18] Cats resistant to SARS-CoV-2 reinfection, News Medical, June 2, 2020.

[19] Tianyue Gao, Xingchen Pan, Chungen Pan, The fate of house cats during the COVID-19 pandemic, Microbes and Infection, 22, 2020, 157.

[20] Margarita Villar, Isabel G. Fernández de Mera, Sara ArtigasJerónimo, Marinela Contreras, Christian Gortázar, and José de la Fuente, Coronavirus in cat flea: findings and questions regarding COVID-19, Parasites \& Vectors, 13, 409, 2020.

[21] Japan Confirms First Cases of COVID-19 in Pets as Two Dogs Test Positive. The Japan Times, August 3, 2020.

[22] Alison E. Stout, Nicole M. André, Javier A. Jaimes, Jean K. Millet, Gary R. Whittaker, Coronaviruses in cats and other companion animals: Where does SARS-CoV-2/COVID-19 fit?, Veterinary Microbiology, 247, 2020, 108777.

[23] Thomas H. C. Sit, Christopher J. Brackman, Sin Ming Ip, Karina W. S. Tam, Pierra Y. T. Law, Esther M. W. To, Veronica Y. T. Yu, Leslie D. Sims, Dominic N. C. Tsang, Daniel K. W. Chu, Ranawaka A. P. M. Perera, Leo L. M. Poon, Malik Peiris, Infection of dogs with SARS-CoV-2, Nature, 586, 2020, 776-778.

[24] N. Oreshkova et al., SARS-CoV2 infection in farmed mink, Netherlands, April 2020. bioRxiv:10.1101/2020.05.18.101493 (18 May 2020). 
[25] Bas B. Oude Munnink, Reina S. Sikkema, David F. Nieuwenhuijse, Robert Jan Molenaar, Emmanuelle Munger, Richard Molenkamp, Arco van der Spek, Paulien Tolsma, Ariene Rietveld, Miranda Brouwer, Noortje Bouwmeester-Vincken, Frank Harders, Renate Hakze-van der Honing, Marjolein C. A. WegdamBlans, Ruth J. Bouwstra, Corine GeurtsvanKessel, Annemiek A. van der Eijk, Francisca C. Velkers, Lidwien A. M. Smit, Arjan Stegeman, Wim H. M. van der Poel, Marion P. G. Koopmans, Transmission of SARSCoV-2 on mink farms between humans and mink and back to humans, Science, Published online November 10, 2020. 